\title{
The Effect of Organizational Characteristics in the Vessel with Foreign Crews on the Self-efficacy and Organizational Commitment
}

\author{
Young-Sub Chung* - Yong-John Shin** -Hoo-Seok Pai*** • †Hyun-Duk Kim**** \\ * CEO, Panstar Shipping, Busan 601-714, Korea \\ ** Assit. Prof., division of Shipping Management, Korea Maritime University, Busan 606-791, Korea \\ *** Special Fellow, Industrial Development Institute, Kyungsung University Busan 608-736, Korea \\ **** Assit. Prof., Dept of Logistics, Sunchon National University, Sunchon 540-742, Korea
}

\begin{abstract}
The primary purpose of this study is to examine empirically the effect of the organizational characteristics in the vessel with foreign crews on their self-efficacy and organization commitment. Currently, the restrictions on the employment of the foreign crews are being eased in the Korean shipping industry and many problems are occurring consequently. However, specific and empirical studies on the relevancy of organizational characteristics to the crew's self-efficacy and organizational commitment on board the vessels with combined crews have not yet been carried out. To solve these problems, we examined how the organizational characteristics such as communication and leadership trust of different language and culture related to vessels with combined crews influence on the self-efficacy, job satisfaction and organizational commitment of the foreign crews through SEM analysis. According to the results, hypothesis 1,2 and 3 were all supported with statistical significance. That means that communication and leadership trust of the vessel with foreign crew had positive effects on their self-efficacy and that such self-efficacy also had a positive effect on the organizational commitment and job satisfaction. To conclude, this study suggests that foreign crew's self-efficacy should be firstly enhanced in order to improve organizational commitment on the vessel with combined crews. Furthermore, studies on self-efficacy of foreign crew's organization are urgently needed to enhance the effectiveness of an organization in a vessel with combined crews that has special environment - working environment with depression and uneasiness due to the exposure to the different language and culture, and excessive stress from the social and psychological adaptation. Therefore, an attempt of this study is considered to be timely since there has not been a study on this subject so far and result of this study will contribute a lot to the organization management of a vessel with combined crews.
\end{abstract}

Key words : Foreign crews, Communication, Leadership trust, Self-efficacy and Organizational commitment

\section{Introduction}

Today, about $65 \%$ in the world merchant fleet has been allowing foreign crews' employment with its own national crews employed in the national fleet and above $10 \%$ of that has been employing foreign crews from above five different countries. In order to improve the international competitive edge of the shipping industry in Korea, the Korean government has been easing limitations to foreigners' employment based on legislative and legal device. Accordingly, the employment of foreign crews has increased to 7 crews. However, organizational characteristics of mixed employment with foreign crews are quite different from that of only national crews' employment. Nonetheless, concrete and empirical study on how such characteristics are related to self-efficacy and organizational commitment as a member boarding a ship has been few. Therefore, the purpose of this study is to test empirically how organizational characteristics such as harmonious communication and trust in leadership are related to self-efficacy of foreign crews, furthermore, and job satisfaction and organizational commitment. The vessel employing foreign crews can enjoy cost saving effect but bring a lot of cultural and organizational problems because mixed crews with different culture and language carry out their job and co-live on board. Recently, effects of human beings' belief, attitude and on organization effectiveness draw a lot of interest. Therefore, much attention are placed on alternatives for being satisfied with job. In particular, role of self-efficacy which can keep anxiety and depression low and overcome situation under stress and motivate themselves with firm confidence and belief about their ability are considered as important. However, individual's self-efficacy of an organization are influenced by working condition and organizational characteristics. The study is to examine empirically the effect of communication and leadership trust of the vessel with foreign crews on their self-efficacy and organizational commitment.

* Representative Author : Young-Sub, Chung, captchung@panstar.biz

** yjshin61@hhu.ac.kr 051) 410-4382

*** hspai@ks.ac.kr 051) 610-0921

+ Corresponding Author : hdkim@sunchon.ac.kr 061)750-5114 
The Effect of Organizational Characteristics in the Vessel with Foreign Crews on the Self-efficacy and Organizational Commitment

\section{Theoretical Background}

Self-efficacy is a belief how much well individual can carry out a certain behaviour at a given situation. Individuals with low self-efficacy tend to fix their target beyond their ability and gap between such high degree of target and self-efficacy raise a negative emotion and put oneself down(Hong, 1995).

Generally, individuals with high self-efficacy show challenging attitude and set a concrete goal. In order to accomplish their goal, they concentrate on due action and attention and also try to solve constantly problems compared with individuals having low self-efficacy when meeting a hard situation and event(Choi, 1997).

According to Toth $(1994)^{\prime} \mathrm{s}$ research result, counsellors with high self-efficacy compared to counsellors with low self-efficacy set a concrete goal and find no difficult in carrying out their counselling job in spite of cultural differences. His research shows that self-efficacy play a important role on the cultural difference's aspects.

In particular, the acceptance and adaptation of different culture have a positive effect on the self-efficacy and also captain's leadership and his communication with crews effect on the self-efficacy (Chung et al., 2006).

The organization management for mixed crews boarding a ship is more difficult compared to the organization of only one nationality's crew boarding a ship because of problems such as difficulty of communication, trust gap in leadership derived from other nationality's officers. The smooth communication and leadership trust effect on self-efficacy of individuals of ship's organization and selt-efficacy have an effect on individual's target level and degree of involvement toward their target and behaviour's selection(Han, 2003; Son, 2001; Choi, 2001).

In other words, foreign crews boarding a ship allowing foreign crews' employment can have more possibility of negative perception due to the environmental factor such as the specific organizational feature and also can negatively influence ship's organization by causing emotional and behavioral response such as stress, maladjustment and switching job. Each organization takes a growing interest in the effect of faith, attitude and emotion of individuals on the organization's efficiency and concentrate on developing a program which can voluntarily carry out its own job and be satisfied with such a job(Shin, 1999).

As mentioned above, It is urgent to study on self-efficacy in ship's organization with foreign crews because it has difficulty in communication, problem of leadership trust and hard stress in social and psychological adaptation coming from different cultural characteristics. And also effects of self-efficacy on organizational commitment must be studied.

\section{The Model, Hypotheses and Measures}

The focal variables in this study are organizational characteristics such as communication and leadership trust, self-efficacy and organization commitment on the vessel with foreign crews. Fig. 1 represents the research model, which reflects the structural relationship or linkage among these variables.

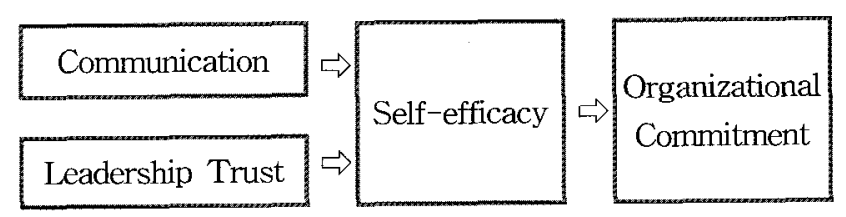

Fig. 1 The Research Model

Three hypotheses have been developed after reviewing a group of prior studies.

Hypothesis 1 : The communication in the vessel with foreign crews will have a positive effect on the self-efficacy.

Hypothesis 2 : The leadership trust in the vessel with foreign crews will have a positive effect on the self-efficacy.

Hypothesis 3 : The self-efficacy of foreign crews will have a positive effect on the organizational commitment.

This study wrote the questionnaire in English. Participants answered using a seven point Likert scale ranging from absolutely disagree to absolutely agree. To measure variables, we selected items from the categories by many earlier studies of organization behavior and psychology.

The degree of smooth communication are measured with a seven point Likert scale by reconstructing items such as organization prospect and integration, individual feedback and communication with seniors and juniors, quality of media, horizontal communication and satisfaction with organizational climate developed by Downs et. al.(1973). And trust in leadership are measured with items such as sincerity, loyalty, senior's fairness and junior's trust developed by Clark and Payne and Niehoff and Moorman respectively. In this study, organizational commitment are measured with organizational involvement and job 
satisfaction. Organizational involvement and job satisfaction are measured wit items used in the Shin's research(1999) selected OCQ and MSQ developed by Mowday, Steers and Porter(1979) and Minnesota university respectively.

\section{Empirical Analysis}

\subsection{Samples and Procedure}

Data were collected from 6 shipping companies in Korea such as Chang Myung Shipping, Panstar Shipping, SK Shipping, STX Pan Ocean, Hanjin Shipping, Hyundai Merchant Marine and 7 overseas crew manning agents in the Philippines, China, Myanmar, Vietnam and Indonesia with questionnaires made by the Likert scales. These collected data were analyzed by reliability analysis, confirmative factor analysis, and structural equation modeling analysis, etc. A total of 700 questionnaires were sent to foreign crews working in these firms from March, 2006 to April, 2006. we obtained a total of 465 responses. Some questionnaires were excluded from the analysis due to missing data. Thus, the sample used for the analysis includes 463 questionnaires.

\subsection{Reliability and Validity}

For reliability of variables, Cronbach's alpha measures how well a set of items (or variables) measures a single unidimensional latent construct. Technically speaking, Cronbach's alpha is not a statistical test - it is a coefficient of reliability (or consistency). Cronbach's alpha can be written as a function of the number of test items and the average inter-correlation among the items. Here, the reliability in this paper is shown to be high using all items because numbers of alpha are high.

Confirmatory factor analysis is used to uncover the latent structure (dimensions) of a set of variables. It reduces attribute space from a larger number of variables to a smaller number of factors and as such is a "non-dependent" procedure (that is, it does not assume a dependent variable is specified). Factor analysis could be used for the purpose to validate a scale or index by demonstrating that its constituent items load on the same factor, and to drop proposed scale items which cross-load on more than one factor.

In this factor analysis, we can see that the rotation sums of squared loadings of factors are more than 1 and these components explain the high percentage of total variance. Therefore, these factors are able to be distinguished, and the conceptual validity is shown because the most of variables' communalities are high.

\subsection{Structural Equation Modeling}

Structural equation modeling (SEM) grows out of and serves purposes similar to multiple regression, but in a more powerful way which takes into account the modeling of interactions, non-linearities, correlated independents, measurement error, correlated error terms, multiple latent independents each measured by multiple indicators, and one or more latent dependents also each with multiple indicators. SEM may be used as a more powerful alternative to multiple regression, path analysis, factor analysis, time series analysis, and analysis of covariance. That is, these procedures may be seen as special cases of SEM, or, to put it another way, SEM is an extension of the general linear model (GLM) of which multiple regression is a part

Advantages of SEM compared to multiple regression include more flexible assumptions (particularly allowing interpretation even in the face of multicollinearity), use of confirmatory factor analysis to reduce measurement error by having multiple indicators per latent variable, the attraction of SEM's graphical modeling interface, the desirability of testing models overall rather than coefficients individually, the ability to test models with multiple dependents, the ability to model mediating variables, the ability to model error terms, the ability to test coefficients across multiple between-subjects groups, and ability to handle difficult data (time series with autocorrelated error, non-normal data, incomplete data).

Indicators are observed variables, sometimes called manifest variables or reference variables, such as items in a survey instrument. Four or more is recommended, three is acceptable and common practice, two is problematic, and with one measurement, error cannot be modeled. Models using only two indicators per latent variable are more likely to be underidentified and/or fail to converge, and error estimates may be unreliable.

Latent variables are the unobserved variables or constructs or factors which are measured by their respective indicators. Latent variables include both independent, mediating, and dependent variables. "Erogenous" variables are independents with no prior causal variable (though they may be correlated with other erogenous variables, depicted by a double-headed arrow -- note two latent variables can be connected by a double-headed arrow (correlation) or a single-headed arrow (causation) but not both. "Endogenous" variables are mediating variables (variables which are both effects of other erogenous or mediating variables, and are 
causes of other mediating and dependent variables), and pure dependent variables. Variables in a model may be "upstream" or "downstream" depending on whether they are being considered as causes or effects respectively. The representation of latent variables based on their relation to observed indicator variables is one of the defining characteristics of SEM. See Table 1.

Table 1 Indicators and latent variables

\begin{tabular}{|c|c|c|}
\hline & 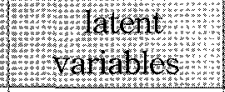 & 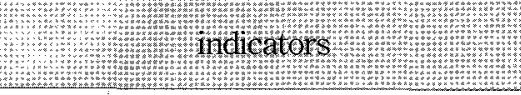 \\
\hline \multirow{2}{*}{ 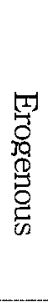 } & $\begin{array}{c}\xi 1: \\
\text { Communication }\end{array}$ & $\begin{array}{l}x_{1}: \text { adequate communication to plan } \\
x_{2}: \text { officials' sufficient advice } \\
x_{3}: \text { full acceptance of task or policy } \\
x_{4}: \text { effort for goal achievement with } \\
\text { communication }\end{array}$ \\
\hline & $\begin{array}{c}\xi 2: \\
\text { Leadership } \\
\text { Trust }\end{array}$ & $\begin{array}{l}x_{5}: \text { loyalty to seniors } \\
x_{6}: \text { sincerity to seniors }\end{array}$ \\
\hline \multirow{2}{*}{ 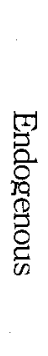 } & \begin{tabular}{c|c}
$n 1:$ & \\
Self-efficacy &
\end{tabular} & $\begin{array}{l}y_{1}: \text { familarity with efficiently } \\
\text { job- performing method } \\
y_{2}: \text { self-confidence in job } \\
y_{3}: \text { job understanding } \\
y_{4}: \text { high performance achievement } \\
y_{5}: \text { ability for overcoming job's barrier }\end{array}$ \\
\hline & $\begin{array}{c}n 2: \\
\text { Organizational } \\
\text { Commitment }\end{array}$ & $\begin{array}{l}z_{1}: \text { involvement } \\
z_{2}: \text { job satisfaction }\end{array}$ \\
\hline
\end{tabular}

The measurement model is that part (possibly all) of a SEM model which deals with the latent variables and their indicators. See Fig. 2.

Structural or Path Coefficients are the effect sizes calculated by the model estimation program. Often these values are displayed above their respective arrows on the arrow diagram specifying a model. In AMOS, these are labeled "regression weights," which is what they are, except that in the structural equation there will be no intercept term. See Table 2.

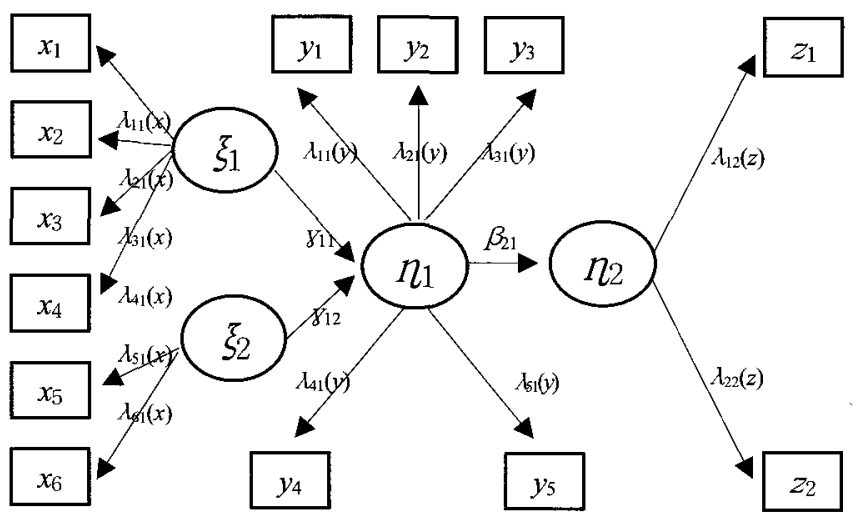

Fig. 2 Path Diagram
When researchers speak of structural or path coefficients in SEM, they normally mean standardized ones. Standardized structural coefficient estimates are based on standardized data, including correlation matrixes. Standardized estimates are used, for instance, when comparing direct effects on a given endogenous variable in a single-group study.

Table 2 Path Coefficients of SEM

\begin{tabular}{|c|c|c|c|c|c|}
\hline 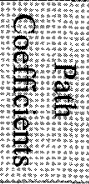 & Estimate & SEC & 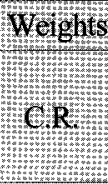 & 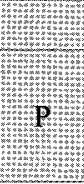 & 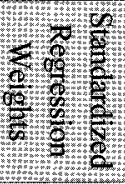 \\
\hline 811 & 0.561 & 0.037 & 15.363 & 0.000 & 0.625 \\
\hline 812 & 0.495 & 0.040 & 12.315 & 0.000 & 0.565 \\
\hline$\beta_{21}$ & 0.873 & 0.040 & 21.994 & 0.000 & 0.935 \\
\hline$\lambda_{11}(x)$ & 1.000 & & & & 0.880 \\
\hline$\lambda_{21}(x)$ & 0.955 & 0.044 & 21.758 & 0.000 & 0.806 \\
\hline$\lambda_{31}(x)$ & 0.960 & 0.039 & 24.376 & 0.000 & 0.862 \\
\hline$\lambda_{41}(x)$ & 0.941 & 0.044 & 21.205 & 0.000 & 0.794 \\
\hline$\lambda_{52}(x)$ & 1.000 & & & & 0.876 \\
\hline$\lambda_{62}(x)$ & 0.985 & 0.069 & 14.324 & 0.000 & 0.769 \\
\hline$\lambda_{11}(y)$ & 1.000 & & & & 0.835 \\
\hline$\lambda_{21}(y)$ & 0.907 & 0.048 & 18.814 & 0.000 & 0.754 \\
\hline$\lambda_{31}(y)$ & 0.916 & 0.047 & 19.618 & 0.000 & 0.776 \\
\hline$\lambda_{41}(y)$ & 0.985 & 0.044 & 22.177 & 0.000 & 0.841 \\
\hline$\lambda_{51}(y)$ & 0.951 & 0.048 & 19.965 & 0.000 & 0.785 \\
\hline$\lambda_{11}(z)$ & 1.000 & & & & 0.897 \\
\hline$\lambda_{21}(z)$ & 0.877 & 0.033 & 26.802 & 0.000 & 0.887 \\
\hline
\end{tabular}

Based the result of this analysis, hypothesis 1 is not rejected because the estimate of standardized regression weights of the path coefficient $\gamma_{11}(0.625)$ is negative and the significant probability is 0.000 . And hypothesis 2 is also not rejected because the estimate of standardized regression weights of the path coefficient $\gamma_{12}(0.565)$ is positive and the significant probability is 0.000 .

Finally, hypothesis 3 is also not rejected because the estimate of standardized regression weights of the path coefficient $\beta_{21}(0.935)$ is positive and the significant probability is 0.000 .

Goodness of fit tests determine if the model being tested should be accepted or rejected. These overall fit tests do not establish that particular paths within the model are significant. If the model is accepted, the researcher will then go on to interpret the path coefficients in the model ("significant" path coefficients in poor fit models are not meaningful). AMOS prints many different goodness-of-fit measures, the choice of which is a matter of dispute among methodologists. Jaccard and Wan (1996) recommend use of at least three fit tests, one from each of the first three categories below, so as to reflect diverse criteria. Kline 
(1998) recommends at least four tests, such as chi-square; GFI, NFI, or CFI; NNFI; and SRMR. Another list of which-to-publish lists chi-square, AGFI, TLI, and RMSEA. There is wide disagreement on just which fit indexes to report, but one should avoid the shotgun approach of reporting all of them, which seems to imply the researcher is on a fishing expedition.

Model chi-square, also called discrepancy, is the most common fit test, printed by all computer programs. The chi-square value should not be significant if there is a good model fit, while a significant chi-square indicates lack of satisfactory model fit. That is, chi-square is a "badness of fit" measure in that a finding of significance means the given model's covariance structure is significantly different from the observed covariance matrix. Generally, if model chi-square $<.05$, the researcher's model is rejected. But, there are three ways, in which the chi-square test may be misleading. Because of these reasons, many researchers who use SEM believe that with a reasonable sample size (ex., $>200$ ) and good approximate fit as indicated by other fit tests (ex., NNFI, CFI, RMSEA, and others), the significance of the chi-square test may be discounted and that a significant chi-square is not a reason by itself to modify the model. See Table 3.

In Table 3, RMR 0.066 is close to significance .05 and GFI 0.865 is also close to significance .90. Futhermore, overall other fit measures(for example, IFI and CFI are more than .90, and NFI, RFI and TLI are close to .90) are considered as "acceptable" in SEM.

Table 3 Fit Measures

\begin{tabular}{|l|r|r|l|}
\hline \multicolumn{1}{|c|}{ Fit Measure } & $\begin{array}{r}\text { Default } \\
\text { model }\end{array}$ & Saturated & Independence \\
Discrepancy & 553.556 & 0.000 & 5496.499 \\
Degrees of freedom & 62 & 0 & 78 \\
P & 0.000 & & 0.000 \\
Number of parameters & 29 & 91 & 13 \\
Discrepancy / df & 8.928 & & 70.468 \\
\hline RMR & 0.066 & 0.000 & 1.453 \\
GFI & 0.865 & 1.000 & 0.166 \\
Adjusted GFI & 0.802 & & 0.027 \\
Parsimony-adjusted GFI & 0.589 & & 0.143 \\
\hline Normed fit index & 0.899 & 1.000 & 0.000 \\
Relative fit index & 0.873 & & 0.000 \\
Incremental fit index & 0.910 & 1.000 & 0.000 \\
Tucker-Lewis index & 0.886 & & 0.000 \\
Comparative fit index & 0.909 & 1.000 & 0.000 \\
\hline
\end{tabular}

\section{Conclusion}

The purpose of this study is to verify empirically the effect of the organizational characteristics of the vessel with foreign crews on their self-efficacy and organization commitment. Currently, the restrictions on the employment of the foreign crews are being eased in the Korean shipping industry and many problems are occurring consequently. However, specific and empirical studies on the relevancy of organizational characteristics to the $\mathrm{crew}^{\prime} \mathrm{s}$ self-efficacy and organizational commitment on board the vessels with combined crews have not yet been carried out. To solve these problems, we examined how the organizational characteristics such as communication and leadership trust of different language and culture related to vessels with combined crews influence on the self-efficacy, job satisfaction and organizational commitment of the foreign crews through SEM analysis.

In conclusion, studies on the self-efficacy of foreign crew organization are urgently needed to enhance the effectiveness of an organization in a vessel with combined crews that has special environment-working environment with depression and uneasiness due to the exposure to the different language and culture, and excessive stress from the social and psychological adaptation. Therefore, an attempt of this study is considered to be timely since there has not been a study on this subject so far and result of this study will contribute a lot to the organization management of a vessel with combined crews. Furthermore, this study will show an empirical analysis output more thoroughly in the making a diagnosis of issues or problems to foreign crews' self efficacy and thus help shipping firms to manage them efficiently.

\section{References}

[1] Choi, C. H. (1997), "The effect of ingroup structure and leader's self-efficacy upon group cohensiveness in initial group counseling sessions", The Graduate School of Keimyung University.

[2] Choi, N. H. and Park, S. J. (2001), "Effects of Motivation to Search and Self - Efficacy related to the WOM Information search on Instrumentality of the WOM Information Source", Korean Society of Consumer Studies, Vol.12 No.2 pp.55-78.

[3] Chung, Y. S., Shin, Y. J., and Pai, H. S. (2006) "The Effect of the Organization Control on the Vessel with Foreign Crews Cultural on the Self-efficacy and Organizational Commitment", Journal of Korea Nation 
The Effect of Organizational Characteristics in the Vessel with Foreign Crews on the Self-efficacy and Organizational Commitment

and Port Research, Vol.30 No.8 pp.643 648.

[4] Clark, M. C. and Payne, R. L. (1997), "The Nature and Structure of Workers' Trust in Management", Journal of Organizational Behavior, 18: 205-24.

[5] Downs, C. W., Hazen, M., Quiggins, J., and Medeley, J. (1973), "An Empirical and Theoretical Investigation of Communication", Paper Presented at the Annual Meeting of the Speech Communication Association, New York.

[6] Han, K. H. (2003), "Self-Efficacy, and Organizational Citizenship Behavior", Daehan journal of business. vol.37 pp. 647-662.

[7] Hong, H. Y. (1995), "The Relationship of Perfectionism, Self-Efficacy and Depression", The Graduate School of Ewha Womans University.

[8] Jaccard, J. and Wan, C. K. (1996), "LISREL approaches to interaction effects in multiple regression". Thousand Oaks, CA: Sage Publications.

[9] Kim, J. K. (2004), "A study on the influences of self-efficacy, motivation and social support on self-esteem", The Graduate School of Hongik University.
[10] Kline, R. B. (1998), "Principles and practice of structural equation modeling". New York: Guilford

[11] Mowday, R., Steers, R., and Porter, L. (1979), "The measurement of Organizational Commitment". Journal of Vocational Behowior, 14, 224-247.

[12] Schindler, P. L. and Thomas, C. C. (1993), "The Structure of Interpersonal Trust in the Workplace", Psychological Reports, 73, 563-573.

[13] Shin Y. J. (1999), "An Empirical Study on the Effect of Organizational Commitment on Consciousness and Behavior of Safety Management". The Graduate School of Changwon University.

[14] Sohn, J. S. (2001), "Effects of Organizational and Personal Characteristics on Salesforces' Performance", Journal of Korean Academy of Marketing science. vol. 8, pp.1-28.

[15] Toth, J. C. (1994), "Basic knowledge asessment tool for critical care nursing, Version 4 (BKAT-4): validity, reliability, and replication". Critical Care Nurse (June), pp. 111-117.

Received 16 February 2007

Accepted 31 March 2007 